\title{
Integration and Evaluation of Spiral Theory based Cybersecurity Modules into core Computer Science and Engineering Courses
}

\author{
Debarati Basu* \\ University of North Carolina at \\ Charlotte \\ Charlotte, North Carolina \\ dbasu1@uncc.edu
N. Dwight Barnette ${ }^{\dagger}$
Virginia Tech
Blacksburg, Virginia
barnette@cs.vt.edu

\author{
Harinni. K. Kumar ${ }^{\dagger}$ \\ Virginia Tech \\ Blacksburg, Virginia \\ kkharinni35@vt.edu
}
Godmar Back ${ }^{\dagger}$
Virginia Tech
Blacksburg, Virginia
gback@vt.edu

\author{
Vinod. K. Lohani ${ }^{\ddagger}$ \\ Virginia Tech \\ Blacksburg, Virginia \\ vlohani@vt.edu
}

\author{
Calvin J. Ribbens ${ }^{\dagger}$ \\ Virginia Tech \\ Blacksburg, Virginia \\ ribbens@vt.edu
}

\author{
Paul E. Plassmann $§$ \\ Virginia Tech \\ Blacksburg, Virginia \\ plassmann@vt.edu
}

\begin{abstract}
Cybersecurity education has been emphasized by several national organizations in the United States, including the National Academy of Engineering, which recognizes securing cyberspace as one of the 14 Engineering Grand Challenges. To prepare students for such challenges and to enhance cybersecurity education opportunities at our large research university, we implemented an NSF-funded cybersecurity education project. This project is a collaborative effort between faculty and graduate students in the Engineering Education, Computer Science (CS) and Computer Engineering (CPE) departments at a major US research university. In this effort, we integrated cybersecurity learning modules into multiple existing core CS and CPE courses following Jerome Bruner's spiral-theory model, which has previously been used to reformulate several academic curricula.

In this paper, we present our cybersecurity curriculum initiative, describe the spiral-theory based process we developed to implement the curriculum and provide an in-depth description of four reusable cybersecurity learning modules that we developed. A core tenet of spiral theory holds to revisit topics as students advance through
\end{abstract}

\footnotetext{
${ }^{*}$ Department of Software and Information Systems

${ }^{\dagger}$ Department of Computer Science

¥Department of Engineering Education

$\S$ Department of Electrical and Computer Engineering
}

Permission to make digital or hard copies of all or part of this work for personal or classroom use is granted without fee provided that copies are not made or distributed for profit or commercial advantage and that copies bear this notice and the full citation on the first page. Copyrights for components of this work owned by others than the author(s) must be honored. Abstracting with credit is permitted. To copy otherwise, or republish, to post on servers or to redistribute to lists, requires prior specific permission and/or a fee. Request permissions from permissions@acm.org.

SIGCSE '20, March 11-14, 2020, Portland, OR, USA

(c) 2020 Copyright held by the owner/author(s). Publication rights licensed to ACM. ACM ISBN 978-1-4503-6793-6/20/03 . \$ \$15.00

https://doi.org/10.1145/3328778.3366798 their curriculum. This work applies this approach to Cybersecurity education by carefully designing the learning objectives of the modules and its contents. For evaluating these learning modules we implemented pre and post-tests to assess students' technical knowledge, their perceptions towards the modules' learning objectives, and how it influenced their motivation to learn cybersecurity. Our findings are overwhelmingly positive and the students' feedback has helped us improve these learning modules. Since its inception, our initiative has educated more than 2000 students and is currently being used to revise the affected courses' syllabi.

\section{CCS CONCEPTS}

- Social and professional topics $\rightarrow$ Model curricula.

\section{KEYWORDS}

curriculum design, cybersecurity education, spiral theory, conceptual learning, multidisciplinary

\section{ACM Reference Format:}

Debarati Basu, Harinni. K. Kumar, Vinod. K. Lohani, N. Dwight Barnette, Godmar Back, Dave McPherson, Calvin J. Ribbens, and Paul E. Plassmann. 2020. Integration and Evaluation of Spiral Theory based Cybersecurity Modules into core Computer Science and Engineering Courses. In The 51st ACM Technical Symposium on Computer Science Education (SIGCSE '20), March 11-14, 2020, Portland, OR, USA. ACM, Portland, OR, USA, 7 pages. https://doi.org/10.1145/3328778.3366798

\section{INTRODUCTION}

The worldwide concern with various cybersecurity attacks results in the need for skilled cybersecurity professionals. In the United States, the National Academy of Engineering recognizes securing cyberspace as one of the 14 Grand Challenges [38]. The National Initiative for Cybersecurity Education (NICE) found in one of their programs that the participants were aware and concerned with 
security issues, but lacked the skill set to protect them from cybercrimes [23]. In 2014, the National Science Foundation organized a cybersecurity education workshop to discuss the opportunities to advance cybersecurity education through educational research and assessment [10]. Since then, many NSF-funded initiatives are being implemented to enhance cybersecurity education $[3,17,22,28,33$, $45,46]$.

This paper discusses an NSF-supported cybersecurity education research that aims to enhance cybersecurity opportunities at the college of engineering at a major US research university. It is a collaborative effort between faculty members and graduate students from the Engineering Education, Computer Science (CS) and Computer Engineering (CPE) departments at this college. In this effort, we adapted a spiral-theory based curriculum model to integrate cybersecurity learning modules at four learning levels in the existing CS and CPE curricula. Its objective is to motivate and prepare undergraduate students starting in their first year to become aware of cybersecurity concepts so that they pursue advanced study in cybersecurity by taking the specialized cybersecurity courses offered at the upper-division level. The project's initial plan was to integrate cybersecurity learning modules in both the CS and the CPE curricula. However, in part due to a concurrent NSF "Revolutionizing Engineering Departments (RED)" grant in CPE that involved other major curriculum changes in that department, we were able to integrate only two of four learning levels at the freshman and sophomore-levels in CPE. Therefore, most of our efforts that we report in this paper were targeted at the CS curriculum. Additionally, this paper presents preliminary findings of the study across all 4 levels.

The paper is organized as follows. First, we discuss the relevant literature on the adaptation of spiral-theory based curricula and existing cybersecurity educational initiatives to emphasize the significance of this work. Second, we illustrate the spiral-theory based cybersecurity curriculum development process that has been iteratively established. Third, to provide specific examples, we present the cybersecurity learning modules implemented in the CS curriculum. Fourth, we explain the purpose and method of cybersecurity education research. Fifth, we report the initial findings of our study evaluating the success of our curriculum modifications. Finally, we discuss the lessons learned, implications and future directions.

\section{RELATED WORK}

\subsection{Spiral Theory-based Curriculum Model}

The concept of a spiral curriculum was proposed by the psychologist Jerome Bruner in his classic works "The Process of Education" [8] and "The Culture of Education" [9] in the twentieth century. In a spiral curriculum model, learners solve authentic problems that increase in complexity at each higher level but are always focused on the same central theme or question. The focus on authentic problems engages novice learners in realistic investigations within a discipline or field of study. An authentic problem should meet multiple criteria: it should be a "doable" problem at the appropriate level for the learner, and at the same time be an open-ended problem that can be expanded in scope, be situated in a social context, resemble real-life practice, and involve active student participation and working as a community where each student has different roles.
Through solving such authentic problems, learners gain sufficient level of mastery over it and the entire conceptual and operational apparatus surrounding it. Bruner's spiral-theory based curriculum model has been successfully adopted to reformulate diverse academic curricula at our school and elsewhere [5, 16, 19, 24, 32]. In this work, we apply a spiral-theory based curriculum model to the domain of cybersecurity education.

\subsection{Cybersecurity Education Initiatives}

Multiple cybersecurity educational initiatives focus on introducing new pedagogical approaches to teach cybersecurity topics. For example, a study evaluated how secure coding and information assurance concepts could be taught to students through a role playing game and related laboratory exercises across various introductory computer courses [1]. A challenge-based learning methodology to motivate undergraduates to solve real-world security problems was implemented at the University of Massachusetts, Boston [14]. A cyberwar lab for undergraduate students to learn penetration-testing techniques and other cybersecurity principles was introduced at Indiana University of Pennsylvania [34] and at the University of Wisconsin [47]. Educators at Syracuse University have been publishing several cybersecurity-related lab exercises [20,21].

Additionally, there have been some studies that presented the development of new undergraduate and graduate cybersecurity courses. For example, a first year cybersecurity course was implemented at the United States Naval Academy [7]. Cyberwar courses have also been implemented at the graduate level [11, 27]. Best practices to design and develop an information security lab course are described in a study by Mattord \& Whitman [36]. Carlson [12] and Mateti [35] discuss the development of cybersecurity courses at Saint Vincent College and Wright State University, respectively. Aman et al. [2] discuss a computer security course that focuses on both offensive and defensive techniques and tools.

Studies that report on the experience with implementing cybersecurity education at the level of an entire program curriculum are fairly limited. Howles et al. [42] show how self-contained reusable cybersecurity modules were created for integration across undergraduate computing curricula. A curricular framework has been developed for undergraduate and graduate programs in information assurance, which is used to develop shared instructional materials, classroom modules and assessment tools [18]. Rowe et al. [41] discuss a framework called "Prepare, Defend, Act" for teaching cybersecurity concepts. Examples of teaching secure coding practices in CS0 and CS1 are discussed in [43, 44]. Although different approaches have been studied to develop and bring cybersecurity learning experiences into the curriculum, to the best of the authors' knowledge no one has attempted the implementation of cybersecurity education using a spiral-based curriculum model in a programmatic way, which is the focus of this work.

Our study also explores students' motivation to learn cybersecurity. Prior studies indicate that students' motivation is positively correlated to their career choices in the future [6, 15, 31]. Past studies also indicate that self-efficacy, one of the constructs of motivation, can vary with gender [40] and ethnicity [26]. The need to investigate self-efficacy of students from various ethnic backgrounds in 
the context of cybersecurity education has been identified by various researchers $[4,13]$. However, there is only a limited number of studies that assess students' motivation to engage in cybersecurity education. To assess student motivation, the MUSIC model was developed as a theoretical model to measure students' motivational change after going through the curriculum [29]. This theoretical framework defines five key constructs related to a student's motivation namely empowerment, usefulness, success, interest and caring. In this study, we focus on the interest and usefulness constructs that indicate a student's belief of how much the modules are interesting or relevant and how the material they are learning in the module will be valuable to their long term goals respectively. This is to investigate if the students are finding the cybersecurity learning modules interesting and useful for their future.

\section{SPIRAL CURRICULUM DEVELOPMENT PROCESS}

Although the cybersecurity spiral curriculum development process was led by the core development team comprised of the authors in this paper, we made sure to include other stakeholders, namely the instructors and graduate student teaching assistants of each of the affected courses. The curriculum was developed iteratively over multiple semesters.

\subsection{Identification of Core Courses}

We identified 4 foundational/core courses in each of the CS and CPE curricula, which all students have to take to meet their respective degree requirements. These four courses, which range from freshman to junior level, represent four learning levels in our curriculum. For example, in CS these courses incluede introductory software design courses as well a sophomore/junior level systems courses as shown in Figure 1. The cybersecurity learning modules developed for each learning level will build on and reinforce learning objectives from the previous, lower level. Although the CS and CPE courses focused on similar topics at each level, they differed in execution (e.g., the departments use different programming languages - CS uses Java/C whereas $\mathrm{CPE}$ uses $\mathrm{C}++$ ), class size, and pedagogical approach.

\subsection{Definition of the Spiral Theme}

Spiral theory emphasizes that if learners participate in an increasingly complex investigation, organized around a central theme or objective that can be repeated at every learning level, they acquire knowledge in a more effective way. The central theme we chose was "Handling threats to software for securing information." Our curriculum is targeted at novices in cybersecurity.

\subsection{Identification of Cybersecurity Concepts}

Based on a review of the relevant literature, we chose the cybersecurity principles outlined in the CIA (Confidentiality, Integrity and Availability) and AAA (Authentication, Authorization and Accounting) models $[25,37]$ as the core concepts to which each level will return.

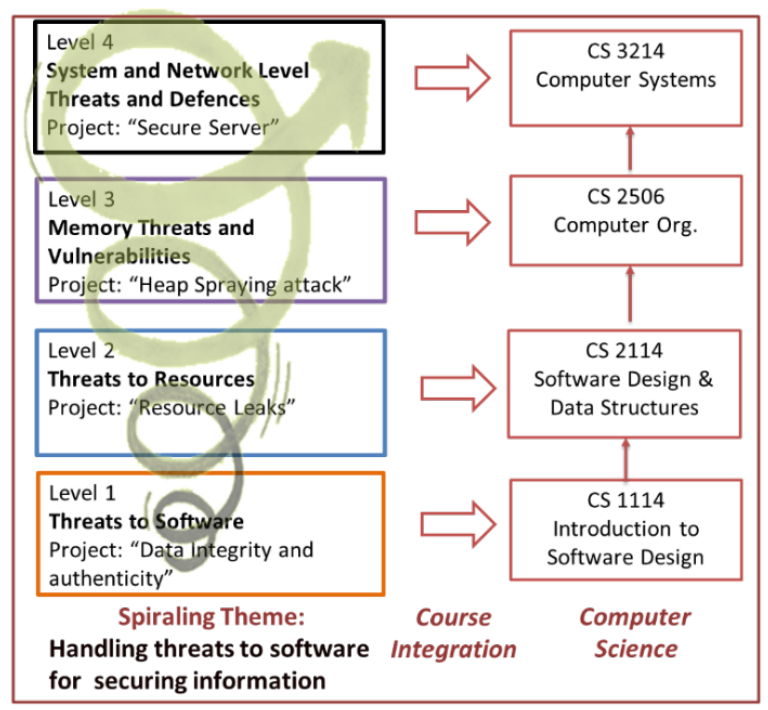

Each level implementation included Lecture Materials and an Authentic Activity

\section{Figure 1: Spiral theory based cybersecurity curriculum}

\section{4 "Spiral"ization of the Cybersecurity Concepts}

Following the selection of the concepts, we mapped each concept to the modules at the four learning levels so that each of the concepts can be revisited in a higher learning level at appropriate depth. The cybersecurity concepts covered at each learning level depended on the content of a particular course in which the cybersecurity learning module would be integrated. For instance, in a data structure design course in which students learn the cost and complexity of data structures, it is natural to relate this discussion to the cybersecurity concept of availability in the face of resource leaks. Figure 2 shows how the concepts has been covered at each level in the CS curriculum. It can be seen that although not all concepts are connected to a project at each level, all concepts are revisited in the higher levels at least once in detail.

\begin{tabular}{|l|l|l|l|l|l|l|}
\hline & Confidentiality & Availability & Integrity & Authenticity & Anonymity & Assurance \\
\hline Level 1 & & & & & & \\
\hline Level 2 & & & & & & \\
\hline Level 3 & & & & & & \\
\hline Level 4 & & & & & & \\
\hline
\end{tabular}

Figure 2: Concepts covered in CS curriculum. Lightly shaded concepts are touched on in lecture; dark shaded concepts are reinforced with an authentic activity.

\subsection{Development of Learning Objectives}

To develop the cybersecurity learning modules at each learning level, learning objectives were established based on the concepts covered in-depth at a particular level. See Table 1 for a list of learning objectives for which we developed learning modules in the respective courses at each level. Since the courses differed in CS 
Table 1 Learning Objectives

\begin{tabular}{|l|l|}
\hline Level & Learning Objectives \\
\hline Level 1 & (1) Define cybersecurity principles: integrity and authenticity \\
(2) Define and explain an adversary model \\
(3) Describe potential security threats from non-validated input \\
(4) Explain the purpose of ensuring the integrity and authentic- \\
ity of data in a real-world scenario \\
(5) Apply the process of ensuring data integrity and authenticity \\
\hline Level 2 & (1) Define cybersecurity principles: data availability and re- \\
(2) Describe potential security threats to resources \\
(3) Identify characteristics of legitimate and malicious requests \\
(4) Apply defense strategy to protect resources \\
\hline Level 3 & (1) Define cybersecurity principles: system integrity and confi- \\
(2) Describe potential security threats to system integrity \\
(3) Design exploits that can compromise system integrity \\
(4) Analyze attack vectors related to unsafe use of memory in \\
Level 4 \\
(3) Determine security threats to web applications and how to \\
(2) Impuages
\end{tabular}

and CPE, their learning modules were also different; however these followed the same learning objectives. The learning objectives were carefully articulated to include words like define, describe, explain, and apply based on Bloom's taxonomy [30] to show the learning outcome designed for each module.

\subsection{Review of Existing Courses}

Since the cybersecurity modules would replace or update existing topics in the non-cybersecurity courses in which they were being integrated, we first reviewed the existing course content with the courses' instructors and course coordinators. The resulting negotiation between the instructor and the team led to the discussion and decision of when and how the module can be integrated into a course. For example, the level 1 CS course (Introduction to Software Design) focuses on designing, implementing, testing, and debugging programs in an object-oriented language. The course was revised to begin exposing students to security as a fundamental aspect of software, in addition to correctness and performance. The cybersecurity learning module focused on illustrating security problems that can occur with input to programs and functions that are not validated.

\subsection{Development of Cybersecurity Learning Modules}

Spiral-based curriculum theory demands that in addition to lecture content, learning modules contain an authentic activity in which students attack a realistic, open-ended problem. Our challenge was to adapt the degree of realism to the learning level and the corresponding expected skill level of the students. This adaptation demands trade-offs in some cases to ensure that the activity fit within the scope of the embedding courses.

\subsection{Implementation and Evaluation of the Learning Modules}

Since the development and implementation of each learning module was quite time consuming, we introduced them in a staggered fashion, starting with Level 1 in the first semester. In each of the following semesters, we introduced one new module at one level and pilot tested it. In the semester after each module was introduced, we revised the module according to the pilot results and carried out an evaluation of its effectiveness.For developing each of the learning modules, we completed each of the steps mentioned in sections 3.5 through 3.8 .

\section{CS CYBERSECURITY LEARNING MODULES}

This section describes the learning modules implemented for the CS courses at each level.

\subsection{Level 1 CS Cybersecurity Learning Module}

For the freshman "Introduction to Software Design" course we developed lecture content and an authentic activity as part of the learning module. The lecture content introduces students to the history of cybersecurity, discusses famous attacks, introduces the CIA/AAA cybersecurity goals and explains the Adversary/Threat model and common infection vectors. In a second lecture, the Alice-Eve-Bob encrypted communication metaphor is introduced as a discussion mechanism for explaining the concepts underlying Diffie-Hellman public key exchange. We introduce one-way hash functions and their use to generate digital signatures.

For the authentic activity, students engaged in a hands-on cybersecurity defense. They were introduced to a specific adversary model that threatens the integrity and authenticity of stored personal data. We assumed that an adversary was able to modify personal user data of a bank, but lacked knowledge of the bank's private encryption keys. To combat this attack, it was explained how the bank would digitally sign the personal data. Students were required to implement a verifier program to check if the adversary had modified the data by checking it against a stored one-way hash that was signed with a private key and verified via the public key. If the data had been tampered with, the electronic signature would fail to certify the content. This project was implemented in Java using the Java Cryptography API. We provided a veneer library for students to use to hide the complexity of Java's cryptography primitives.

\subsection{Level 2 CS Cybersecurity Learning Module}

In the module created for the sophomore "Software Design \& Data Structures" course, we reintroduced students to the CIA/AAA cybersecurity goals. We introduced denial of service (DOS) attacks, focusing on cache flooding as a particular attack vector threatening the goals of availability and resource integrity in a system. The activity is split into two labs. The first lab introduces the threat; students develop a defense in the second lab. These labs were designed 
to complement existing lab activities in this course, which were focused on the design and use of container data structures such as linked lists and hash tables. The module involves a cache container data structure used to store and retrieve the data returned by a service in response to client requests. When subjected to an adversarial sequence of requests, the container could grow unbounded unless cache resource management techniques are deployed. This results in decreased availability; an example of a classic DOS attack.

In the first lab students complete the implementation of an inmemory linear container data structure, learning about linked lists in the process. Then students submit their linear cache container to a back end testing system where it is used as the cache component for a server. The testing system integrates the student's linear container into a system to act as a cache and executes multiple input data sets upon it, representing both common (benign) and attack workloads. The benchmark performance results are presented to the students via a web-based interface, who analyze the results to learn how common and attack workloads affect system availability and integrity. In the second lab, students must deal with one cache workload where 90 percent of the cache request stream is generated by an attacker attempting to flood the cache by generating sequential unique accesses that lack locality. The students implement a least recently used (LRU) cache eviction policy by incorporating a hash data structure for fast lookup, and use the linear linked-list data structure to find the least-recently-used data in the cache to replace. Once again the students submit their implemented cache to a back end testing system which generates attack performance results for the students to analyze. Student can iterate, and are shown the throughput and latency of a server employing their concrete data structure implementation would provide. When successful, the students will have learned how to implement and apply a defense strategy to achieve data availability and resource integrity even in the face of realistic cyber attacks.

\subsection{Level 3 CS CyberSecurity Learning Module}

The learning module introduces in the sophomore level systems course "Computer Organization" focused on memory threats and vulnerabilities, which are related to the cybersecurity concepts of integrity and confidentiality. The main content of this course focuses on processor design and instruction set architectures. Students learn and practice assembly programming and understanding how native code is executed by a processor. The cybersecurityrelated authentic activity that we introduced via our module was a heap spraying attack.

Heap spraying is a method in which an attacker uses existing memory allocation facilities to place snippets of malicious code into the victim's memory, then trigger buffer overflow vulnerability in an attempt to make the victim execute said code. Heap spraying attacks represent a successor generation to simple buffer overflow attacks in which an attacker places code on the victim's executable stack. For our project, we assumed a web browser with said vulnerability. Students apply their knowledge of instruction set architectures and system call conventions to produce machine code that executes the attack, then they write JavaScript code to place the attack payload into the victim's heap. The resulting project realistically duplicates the steps an attacker might take. We implemented this project using the v8 JavaScript engine embedded in Node.js, simulating the environment found in browser employing this engine. Students submit JavaScript code to our web-based grading interface in a form similar to how an attacker might try to place malicious JavaScript code into third-party JavaScript libraries. The interface reports back to the students whether the attack was successful in executing remote code (in this case, an executable ./success).

\subsection{Level 4 CS CyberSecurity Learning Module}

Level 4 was implemented in the junior-level "Computer Systems" course. The main goal of level 4 was to emphasize the importance of cybersecurity in the context of web applications. The cybersecurity concepts of authenticity, anonymity, and confidentiality were discussed in depth through lectures and students gained practice with a newly designed project that represented the authentic activity. The lectures included a brief overview of the cybersecurity concepts, Transport Layer Security (TLS) and different authentication methods. TLS was reviewed to see how it addresses confidentiality, integrity, and authenticity in client-server communication. To emphasize the concept of authenticity, stateful and stateless authentication methods were explored. JWT (JSON web tokens) as defined in RFC 7519 were introduced as a mechanism to implement stateless authentication.

For the accompanying projects, students implemented a small personal web server, which can serve some common files as well as provide access to a user-specific secure part of the server which is contingent on user authentication. The project consisted of two parts. First is a $\mathrm{C}$ language-based implementation of basic web server functionality, including an implementation of the HTTP 1.1 protocol. In the second part, student apply the cybersecurity concepts they have studied by implementing a stateless authentication via JWT to authenticate the users. This involves the steps of authentication (via username and password), the creation of a signed JWT token with expiration date, and returning said token to the requesting client. On subsequent requests, the token will be presented by the client and must be checked by the server for validity based on a digital signature and whether the token has expired. We created a test harness to test students' implementation for robustness under a multitude of scenarios, including scenarios in which clients misbehave in a number of ways, for instance, by presenting corrupted or expired tokens. Thus, through this activity, students gained sufficient skills to develop a web application that ensures authenticity and confidentiality. In the context of the course, this project replaced an older version which did not include any cybersecurity aspects.

\section{EVALUATION AND RESULTS}

The purpose of the evaluation was to investigate students' gain in conceptual knowledge and motivation in cybersecurity concepts after the implementation of the learning modules in the respective courses. For each learning level, a pilot test was developed to perform the evaluation. After pilot testing a learning module in a particular semester, we refined it the following semester based on feedback from the students. Then in the subsequent semester, a pre-test and a post-test surveys were conducted to capture the change in students' knowledge and motivation due to the learning 
Table 2 Affected Student Demographics

\begin{tabular}{|c|c|c|c|c|c|}
\hline Learning Levels & $\begin{array}{l}\text { Courses (Total } \\
\text { \# of Students) }\end{array}$ & Sample size $\mathrm{n}$ & Academic Level & Gender & Ethnicity \\
\hline Level 1 & $\begin{array}{l}\text { CS1114 (385) } \\
\text { ECE1574 (370) }\end{array}$ & $\begin{array}{l}\text { CS1114 (54) } \\
\text { ECE1574 (78) }\end{array}$ & $\begin{array}{l}\text { Freshman: } 90 \\
\text { Sophomore: } 22 \\
\text { Junior: } 12 \\
\text { Senior: } 8\end{array}$ & $\begin{array}{l}\text { Female: } 29 \\
\text { Male: } 101 \\
\text { Others: } 2\end{array}$ & $\begin{array}{l}\text { White/Caucasians: } 77 \\
\text { Others: } 55\end{array}$ \\
\hline Level 2 & $\begin{array}{l}\text { CS2114 (270) } \\
\text { ECE2574 (64) }\end{array}$ & $\begin{array}{l}\text { CS2114 (123) } \\
\text { ECE2574 (41) }\end{array}$ & $\begin{array}{l}\text { Freshman: } 14 \\
\text { Sophomore: } 101 \\
\text { Junior: } 40 \\
\text { Senior: } 9\end{array}$ & $\begin{array}{l}\text { Female: } 39 \\
\text { Male: } 124 \\
\text { Others: } 1\end{array}$ & $\begin{array}{l}\text { White/Caucasians: } 94 \\
\text { Others: } 70\end{array}$ \\
\hline Level 3 & CS2506 (155) & CS2506 (105) & $\begin{array}{l}\text { Sophomore: } 36 \\
\text { Junior: } 59 \\
\text { Senior: } 10\end{array}$ & $\begin{array}{l}\text { Female: } 15 \\
\text { Male: } 90\end{array}$ & $\begin{array}{l}\text { White/Caucasians: } 56 \\
\text { Others: } 49\end{array}$ \\
\hline Level 4 & CS3214 (160) & CS3214 (120) & $\begin{array}{l}\text { Junior: } 87 \\
\text { Senior: } 33\end{array}$ & $\begin{array}{l}\text { Female: } 28 \\
\text { Male: } 92\end{array}$ & $\begin{array}{l}\text { White/Caucasians: } 64 \\
\text { Others: } 56\end{array}$ \\
\hline
\end{tabular}

modules. The survey included questions in five categories: a) demographic questions including gender identification, academic level, discipline and ethnicity, b) 5-point Likert scale questions (strongly disagree to strongly agree) to understand students' perceptions towards the modules' learning objectives, c) multiple-choice conceptual questions focused on each learning objective of a module, $d$ ) 6-point Likert scale questions (strongly disagree to strongly agree) to understand students' perceptions on whether they found the cybersecurity modules useful and interesting (adapted from the MUSIC model [29]), and e) open-ended questions for obtaining students' feedback about the learning modules.

Levels 1 and 2 were implemented in CS and CPE courses while levels 3 and 4 were implemented in CS courses only. Table 2 provides demographic information of the participants of the study.

While the analysis of the data collected across the levels are still underway, the initial quantitative results of the evaluation are presented in Table 3. For each learning module, we asked students during a pre-test and a post-test to assess their level of confidence with respect to each of the learning objectives of a module. The second and third column indicate the aggregate mean and standard deviation for the level of confidence value for each of the learning levels from the pre and post tests, respectively. A paired t-test was conducted to check if the difference between the mean pre-test and post-test values was statistically significant. The $\mathrm{p}$-values of the t-tests are presented in column four. The results show that students' level of confidence with respect to the learning objectives of the modules significantly increased from pre to post-tests for all the four learning levels due to the implementation of the cybersecurity modules.

Further, an ANOVA test was carried out to determine if the difference between the mean pre-test and post-test values differed significantly by academic levels, gender or ethnicity. The p-values for these tests are noted in the last three columns. The p-values with three and two stars $\left(^{*}\right)$ represent 0.01 and 0.05 level of significance, respectively. It is found that except for the females of level 1, who had a significantly higher level of confidence with the learning objectives compared to males, the confidence level did not significantly differ in terms of gender, and ethnicity. It was interesting to observe that the seniors, who took level 4 seemed to have a higher agreement than juniors in this evaluation category. However, overall there is not much difference across student groups about their level of confidence with the learning objectives of the cybersecurity modules. This shows that according to the students'
Table 3: Results for students' level of confidence with the learning modules across all levels

\begin{tabular}{|c|c|c|c|c|c|c|}
\hline \multirow{2}{*}{$\begin{array}{l}\text { Learning } \\
\text { Levels }\end{array}$} & \multirow{2}{*}{$\begin{array}{l}\text { Mean } \\
\text { Pre-Test } \\
\text { Value }\end{array}$} & \multirow{2}{*}{$\begin{array}{l}\text { Mean } \\
\text { Post-Test } \\
\text { Values }\end{array}$} & \multirow{2}{*}{$\begin{array}{l}\text { p-value for } \\
\text { change from } \\
\text { pre- to } \\
\text { post-test }\end{array}$} & \multicolumn{3}{|c|}{$\mathrm{p}$-values based on various factors } \\
\hline & & & & $\begin{array}{c}\text { Academic } \\
\text { Level }\end{array}$ & Gender & Ethnicity \\
\hline Level 1 & $2.99(1.23)$ & $4.29(0.66)$ & $\begin{array}{l}<0.0001^{* * *} \\
(\text { pre }<\text { post) }\end{array}$ & 0.6677 & $\begin{array}{l}0.0284^{* *} \\
\text { (female } \\
>\text { male) }\end{array}$ & 0.4789 \\
\hline Level 2 & $3.15(1.06)$ & $4.06(0.94)$ & $\begin{array}{l}<0.0001^{* * *} \\
(\text { pre }<\text { post })\end{array}$ & 0.0588 & 0.6836 & 0.5494 \\
\hline Level 3 & $2.54(0.93)$ & $3.17(0.97)$ & $\begin{array}{l}<0.0001 \\
(\text { pre }<\text { post) }\end{array}$ & 0.4366 & 0.7934 & 0.1087 \\
\hline Level 4 & $2.98(0.97)$ & $3.38(0.80)$ & $\begin{array}{l}<0.0001^{* * *} \\
\text { (pre<post) }\end{array}$ & $\begin{array}{c}0.03802 \\
\text { (senior> } \\
\text { junior) }\end{array}$ & 0.5799 & 0.5213 \\
\hline
\end{tabular}

opinion, we were able to meet the cybersecurity learning objectives for all the diverse group of students.

\section{CHALLENGES, SUSTAINABILITY AND FUTURE DIRECTIONS}

Implementing a curriculum initiative across multiple departments provided multiple challenges. First, it ran into conflicts with a simultaneous curriculum reorganization as part of the NSF-Revolutionizing Engineering Department efforts, causing us to abandon the implementation of learning levels 3 and 4 in one department. Second, the substantial enrollment growth in both CS and CPE concurrent with the implementation of our project imposed a significant burden on the instructors in all courses, limiting their bandwidth available for modifying existing course content. Third, the introduction of each module required substantial Graduate Teaching Assistants (GTA) support to help implement and maintain infrastructure, which was partly funded by matching in-kind contributions from each department. We found it difficult to recruit GTAs with the specialized cybersecurity background we needed. In addition, GTA positions are often only one or two semesters long as students transition to GRA positions, which made it difficult to build and retain institutional knowledge during the introduction phase of our new curriculum. Fourth, faculty engagement in the project has been challenging for some courses and some semesters. Some classes are taught by a rotating group of faculty instructors, not all of which are equally invested in the course. On a related note, we needed to convince the course coordinators, who have a long term interest in the course to view the modules as an enrichment and improvement to their existing course structures that they were willing to accept. To sustain these changes brought about by our initiative, we formalized the learning objectives of each of the course's modules within each course's official syllabus.

This paper presents the design and implementation process to incorporate cybersecurity learning modules into existing foundational courses in the CS curriculum based on spiral theory. We plan to carry out and report further analysis results in a subsequent paper. Our results so far indicate that the students agree that the cybersecurity learning objectives were met. The results of this project, including the learning modules themselves, are being made available via a website [39] for adoption at other institutions. 


\section{REFERENCES}

[1] N. Adamo-Villani, M. Oania, and S. Cooper. 2012. Using a Serious Game Approach to Teach Secure Coding in Introductory Programming: Development and Initial Findings. Fournal of Educational Technology Systems 41, 2 (2012), 107-131. https: //doi.org/10.2190/ET.41.2.b

[2] J. Aman, J.E. Conway, and C. Harr. 2010. A capstone exercise for a cybersecurity course. Fournal of Computing Sciences in Colleges 25 (2010), 207-212.

[3] M. Anazco, A. J. Magana, and B. Yang. 2016. Employing Model-Eliciting Activities in Cybersecurity Education. In ASEE Annual Conference \& Exposition. ASEE, New Orleans, LA. https://peer.asee.org/26943.

[4] S. Bagchi-Sen, H.R. Rao, J.S. Upadhyaya, and S. Chai. 2010. Women in Cyber security: A Study of Career Advancement. IT Professional 12 (03 2010), 24-31. https://doi.org/10.1109/MITP.2010.39

[5] Ganesh Balasubramanian, Vinod Lohani, Ishwar Puri, Scott W Case, and Roop Mahajan. 2011. Nanotechnology EducationâĂŤFirst Step in Implementing a Spiral Curriculum*. The International fournal of Engineering Education 27 (01 2011).

[6] A. Bandura, C. Barbaranelli, G. Caprara, and C. Pastorelli. 2001. Self-Efficacy Beliefs as Shapers of Children's Aspirations and Career Trajectories. Child Development 72 (01 2001), 187-206. https://doi.org/10.1111/1467-8624.00273

[7] C. Brown, F. Crabbe, R. Doerr, R. Greenlaw, C.Hoffmeister, J. Monroe, D. Needham, A. Phillips, S. Schall A. Pollman, J. Schultz, S. Simon, D. Stahl, and S. Standard 2012. Anatomy, Dissection, and Mechanics of an Introductory Cyber-security Course's Curriculum at the United States Naval Academy. In Proceedings of the 17th ACM Annual Conference on Innovation and Technology in Computer Science Education (ITiCSE '12). ACM, Haifa, Israel, 303-308. https://doi.org/10.1145/ 2325296.2325367

[8] J. S. Bruner. 1960. The process of education. Harvard University Press, Cambridge, MA, USA.

[9] J. S. Bruner. 1996. The culture of education. Harvard University Press, Cambridge, MA, USA.

[10] S. Buck and D. Burley. 2014. Cybersecurity education workshop: Final report. Technical Report. George Washington University, Arlington, VA, USA.

[11] R. Doerr C. Brown, F. Crabbe and R. Greenlaw. 2003. Teaching hands-on network security: Testbeds and live exercises. FIW 3, 2 (2003), 8-25.

[12] D. Carlson. 2004. Teaching Computer Security. SIGCSE Bulletin 36, 2 (June 2004), 64-67. https://doi.org/10.1145/1024338.1024374

[13] S. Chai, S. Bagchi-Sen, R. Goel, H. Raghav Rao, and J.S. Upadhyaya. 2006. A Framework for Understanding Minority Students' Cyber Security Career Interests. In Proceedings of the 12th Americas Conference On Information Systems, Vol. 6. AMCIS, Atlanta, GA,USA, 413.

[14] R.S. Cheung, J.P. Cohen, H.Z. Lo, and F. Elia. 2011. Challenge Based Learning in Cybersecurity Education. Presented at the International Conference on Security and Management. http://worldcomp-proceedings.com/proc/p2011/SAM5063.pdf

[15] D.E. Chubin, K.M. Donaldson, B. Olds, and L.N. Fleming. 2008. Educating Gen eration Net-Can U.S. Engineering Woo and Win the Competition for Talent? fournal for Engineering Education 97(3) (2008), 245-258.

[16] A. G. Dixon D. DiBiasio, L. Comparini and W. M. Clark. 2001. A Project-based Spiral Curriculum for Introductory Courses in ChE: Part 3. Evaluation. Chemical Engineering Education,2001 35(2) (2001), 140-47.

[17] A. Das, C. Choi D. Voorhees, and C.E. Landwehr. 2017. Cybersecurity for Future Presidents: An Interdisciplinary Non-majors Course. In Proceedings of the 2017 ACM SIGCSE Technical Symposium on Computer Science Education (SIGCSE '17). ACM, Seattle, WA, USA, 141-146. https://doi.org/10.1145/3017680.3017713

[18] J. Davis and M. Dark. 2003. Defining a curriculum framework in information assurance and security. In Proceedings of the 2003 ASEE Annual Conference. ASEE.

[19] A. Dixon, W. Clark, and D. Dibiasio. 2000. Project-based, spiral curriculum for introductory courses in ChE. Part 2. Implementation. Chemical Engineering Education,2000 34(4) (09 2000), 296-303.

[20] W. Du. 2011. SEED: Hands-On Lab Exercises for Computer Security Education. IEEE Security Privacy 9, 5 (2011), 70-73. https://doi.org/10.1109/MSP.2011.139

[21] W. Du and R. Wang. 2008. SEED: A Suite of Instructional Laboratories for Computer Security Education. FERIC 8, 1, Article 3 (March 2008), 24 pages. https://doi.org/10.1145/1348713.1348716

[22] K. Fisle, C. Shue, J. Guttman, and K. Kumar. 2015. SaTC-EDU: EAGER: Enhancing Cybersecurity Education through Peer Review. NSF award number 1500039n at Worcester Polytechnic Institute,. https://www.nsf.gov/awardsearch/showAward? AWD ID $=1500039$

[23] S. Furman, Yee-Yin M. F. Theofanos, Y-Y. Choong, and Brian B. Stanton. 2012. Basing Cybersecurity Training on User Perceptions. IEEE Security and Privacy 10, 2 (2012), 40-49. https://doi.org/10.1109/MSP.2011.180

[24] K.V. Gupta, B. Joseph, N. Alcantar, R. Toomey, and A. Sunol. 2008. A Spiral Curriculum for Chemical Engineering. AIChE Annual Meeting, Conference Proceedings 54 (11 2008).

[25] Hack2Secure. 2018. An Introduction to Core Security Concepts CIA Triad And AAA. https://www.hack2secure.com/blogs/an-introduction-to-core-securityconcepts-cia-triad-and-aaa
[26] G. Hackett, N. Betz, C. Manuel, and A.I. Rocha-Singh. 1992. Gender, Ethnicity, and Social Cognitive Factors Predicting the Academic Achievement of Students in Engineering. Fournal of Counseling Psychology 39 (10 1992), 527-538. https: //doi.org/10.1037/0022-0167.39.4.527

[27] J.M.D. Hill, C.A. Carver, J.W. Humphries, and U.W Pooch. 2001. Using an Isolated Network Laboratory to Teach Advanced Networks and Security. In Proceedings of the Thirty-second SIGCSE Technical Symposium on Computer Science Education (SIGCSE '01). ACM, NC, USA, 36-40. https://doi.org/10.1145/364447.364533

[28] G. Richard I. Ahmed, V. Roussev. 2015. SaTC-EDU: EAGER: Peer Instruction for Cybersecurity Education. NSF award number 1500101 at University of New Orleans. https://www.nsf.gov/awardsearch/showAward?AWD ID=1500101

[29] B. Jones. 2009. Motivating students to engage in learning: The MUSIC Model of Academic Motivation. International fournal of Teaching and Learning in Higher Education 21 (01 2009), 272-285.

[30] D. R. Krathwohl. 2002. A Revision of Bloom's Taxonomy: An Overview. Theory Into Practice 41, 4 (2002), 212-218. https://doi.org/10.1207/s15430421tip4104_2

[31] W.R. Lent and G. Hackett. 1987. Career self-efficacy: Empirical status and future directions. Journal of Vocational Behavior 30 (06 1987), 347-382. https://doi.org/ 10.1016/0001-8791(87)90010-8

[32] Vinod K. Lohani, Mary Leigh Wolfe, Terry Wildman, Kumar Mallikarjunan, and Jeffrey Connor. 2010. Reformulating general engineering and biological systems engineering programs at Virginia Tech. Advances in Engineering Education 2, 4 (1 12 2010), 1-30.

[33] A. Shniderman M. Bachmann, L. Solberg. 2015. SaTC-EDU: EAGER: Education Initiative TECH MeD: Transdisciplinary Education for Critical Hacks of Medical Devicess. NSF award number 1500077 at Texas Christian University. https: //www.nsf.gov/awardsearch/showAward?AWD_ID $=1500077$

[34] M. Mary and H. Rossman. 2002. Building a Cyberwar Lab: Lessons Learned: Teaching Cybersecurity Principles to Undergraduates. SIGCSE 34, 1 (2002), 23-27. https://doi.org/10.1145/563517.563349

[35] P. Mateti. 2003. A Laboratory-based Course on Internet Security. SIGCSE Bulletin 35, 1 (Jan. 2003), 252-256. https://doi.org/10.1145/792548.611982

[36] H.J. Mattord and M.E. Whitman. 2004. Planning, Building and Operating the Information Security and Assurance Laboratory. In Proceedings of the 1st Annual Conference on Information Security Curriculum Development (InfoSecCD '04). ACM, Kennesaw, GA, USA, 8-14. https://doi.org/10.1145/1059524.1059527

[37] L. Nweke. 2017. Using the CIA and AAA Models to explain Cybersecurity Activities. https://pmworldlibrary.net/wp-content/uploads/2017/05/171126Nweke-Using-CIA-and-AAA-Models-to-explain-Cybersecurity.pdf

[38] National Academy of Engineering. 2017. Grand Challenges for Engineering. Washington, DC. http://www.engineeringchallenges.org/9042.aspx

[39] CyberSecurity Education project. 2019. An NSF Funded CyberSecurity Education project at VT. Virginia Tech. https://hosting.cs.vt.edu/CybersecurityEducation

[40] M.B. Rosson, J.M. Carroll, and H. Sinha. 2011. Orientation of Undergraduates Toward Careers in the Computer and Information Sciences: Gender, Self-Efficacy and Social Support. TOCE 11, 3, Article 14 (2011), 23 pages. https://doi.org/10. $1145 / 2037276.2037278$

[41] D.C. Rowe, B.M. Lunt, and J. Ekstrom. 2011. The Role of Cyber-security in Information Technology Education. In Proceedings of the 2011 Conference on Information Technology Education (SIGITE '11). ACM, West Point, NY, USA, 113122. https://doi.org/10.1145/2047594.2047628

[42] S. Mishra Sumita T. Howles, C. Romanowski and R.K. Rajendra. 2011. A holistic, modular approach to infuse cybersecurity into undergraduate computing degree programs. In Annual Symposium On Information Assurance (ASIA), Albany, NY. $7-8$.

[43] B. Taylor, M. Bishop, D. Burley, S. Cooper, R. Dodge, and R. Seacord. 2012. Teaching Secure Coding: Report from Summit on Education in Secure Software. In Proceedings of the 43rd ACM Technical Symposium on Computer Science Education (SIGCSE '12). ACM, Raleigh, NC, USA, 581-582. https://doi.org/10.1145/2157136. 2157304

[44] B. Taylor and S. Kaza. 2016. Introducing Secure Coding in CS0, CS1, and CS2 (Abstract Only). In Proceedings of the 47th ACM Technical Symposium on Computing Science Education (SIGCSE '16). ACM, Memphis, TN, USA, 715-715. https://doi.org/10.1145/2839509.2844684

[45] D. Voorhees, A. Das, and C. Choi. 2017. Injecting and Assessing Cybersecurity Topics Within a Computer Science Program. Fournal of Computing Sciences in Colleges 32, 6 (June 2017), 54-66. http://dl.acm.org/citation.cfm?id=3069658. 3069670

[46] Y. Sakamoto W. H. Hui, Y. Tao. 2015. SaTC-EDU: EAGER: Development and Evaluation of Privacy Education Tools via Open Collaboration. NSF award number 1464800 at Stevens Institute of Technology, NJ, USA. https://www.nsf.gov/ awardsearch/showAward?AWD_ID $=1464800$

[47] P.J. Wagner and J.M. Wudi. 2004. Designing and Implementing a Cyberwar Laboratory Exercise for a Computer Security Course. In Proceedings of the 35th SIGCSE Technical Symposium on Computer Science Education (SIGCSE '04). ACM, VA, USA, 402-406. https://doi.org/10.1145/971300.971438 